\title{
Reversible Photon-Induced Oxidation of Graphene by $\mathrm{NO}_{2}$ Adsorption
}

\author{
Stefan Böttcher*, Hendrik Vita, Karsten Horn \\ Fritz-Haber Institute of the Max-Planck Society, Faradayweg 4-6, 14195 Berlin, Germany
}

\begin{abstract}
We show that an oxidized graphene sheet is formed by $\mathrm{NO}_{2}$ adsorption and subsequent photodissociation, through an analysis of X-ray absorption and photoemission spectroscopic results. Adsorption of $\mathrm{NO}_{2}$ on graphene grown on $\operatorname{Ir}(111)$ leads to a strong rehybridization of the unoccupied $\pi^{*}$ states and the appearance of new features in the $\mathrm{C} 1 s$ core level line. This is a reversible process, such that pristine graphene can be recovered by thermal treatment. The formation of oxidized graphene is sensitive to the photon-flux, resulting in an intermediate phase of oxidized graphene. Our results provide an interesting pathway towards lithographic patterning processes in graphene.
\end{abstract}

Keywords: Graphene, Graphene Oxide, Functionalization, NEXAFS, XPS, Adsorbates

\section{Introduction}

The influence of ambient species is of great importance for the widespread use of graphene in technology, and this topic has thus received considerable attention in recent years [1-5]. Many molecules exhibit only a weak adsorption bond to graphene; the polar molecules, $\mathrm{H}_{2} \mathrm{O}$ and $\mathrm{NH}_{3}$ may serve as examples $[6,7]$. As a consequence, they are molecularly adsorbed with no sign of dissociation, not even under the influence of ultraviolet (UV) radiation. Molecular oxygen itself is reported to only intercalate between graphene and $\operatorname{Ir}(111)$ with no sign of chemical interaction [8]. $\mathrm{NO}_{2}$, on the other hand, is more strongly adsorbed, as judged from experimental data for adsorption on carbon nanotubes [9], and its dissociation under photon irradiation is well known [10-12]. The preparation of oxidized graphene sheets has so far been achieved by either a strong physical or chemical treatment such as oxygen atom bombardment [13], or dispersion of graphite oxide in basic solutions, yielding what is expected to be a precursor to graphene preparation [14]. Considering this precursor application of oxidized graphene (we will refer to this in the following as graphene oxide, GrO), the quality of the GrO layer has been reported to be essential for the thermal recovery of graphene [15]. The authors show that the thermal recovery of graphene reveals the highest quality for low oxygen coverages in $\mathrm{GrO}$, consisting of mainly epoxidic oxygen atoms, while other oxide phases destroy the graphene backbone. Here we provide an alternative and less intrusive pathway for $\mathrm{GrO}$ preparation, by $\mathrm{NO}_{2}$ adsorption and subsequent dissociation under UV irradiation, which induces a transformation of graphene into GrO, consisting of mainly the favored epoxidic oxygen atoms and fewer defects on graphene. We explain the observed results in the context of a simple photon-induced

\footnotetext{
${ }^{*}$ Corresponding Author Tel.: +49 3084135620

Email address: boettcher@fhi-berlin.mpg.de (Stefan Böttcher)
}

oxidation mechanism.

\section{Experimental Details}

The photoemission spectroscopy (PES) and near-edge x-ray absorption fine structure (NEXAFS) measurements were performed at the UE56/2-PGM-1 and PGM-2 beamlines of the BESSY II synchrotron radiation facility, Berlin (Germany). Graphene was prepared on a clean $\operatorname{Ir}(111)$ single crystal surface. The cleaning process consisted of subsequent cycles of thermal flashing up to $1800^{\circ} \mathrm{C}$ followed by annealing at an oxygen partial pressure of $1 \times 10^{-6} \mathrm{mbar}$ at $1300^{\circ} \mathrm{C}$. Graphene on $\operatorname{Ir}(111)$ was prepared by thermal decomposition of ethylene at $1250^{\circ} \mathrm{C}$ at a partial pressure of $1 \times 10^{-6}$ mbar. The quality of the graphene/Ir(111) sample was verified by means of low energy electron diffraction (LEED) and core-level as well as valenceband photoelectron spectroscopy. The adsorption of high purity $\mathrm{NO}_{2}$ was performed at a partial pressure of $p=5 \times 10^{-8} \mathrm{mbar}$ and a sample temperature of $100 \mathrm{~K}$. The temperature was kept constant for all following measurements, unless declared otherwise. NEXAFS spectra were collected at the carbon $K$ and oxygen $K$ absorption edges in the surface sensitive partial electron yield mode (PEY) using a hemispherical detector and Auger electron yield. The base pressure during measurements was better than $7 \times 10^{-11}$ mbar.

\section{Results and Discussion}

Figure 1 shows a set of photoemission (PE) survey spectra of graphene/ $\operatorname{Ir}(111)$, starting with the pristine graphene surface and stepwise exposure of $\mathrm{NO}_{2}$ up to 1400 Langmuir, recorded at $800 \mathrm{eV}$ photon energy and an average flux of approx. $10^{11}$ photons/sec and a total photon dose of approx. $10^{14}$ photons. 


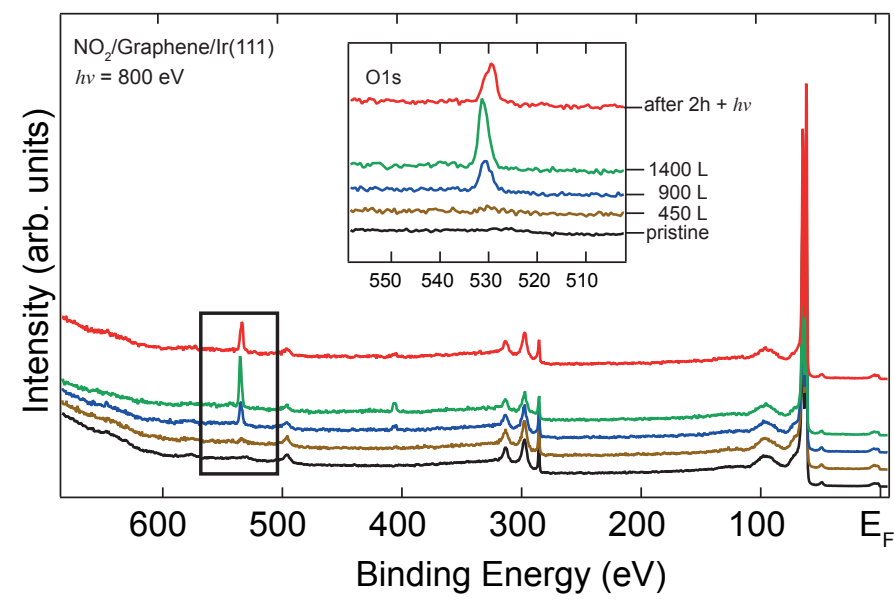

Figure 1: PE overview spectra for the $\mathrm{NO}_{2}$ deposition on graphene/ $\operatorname{Ir}(111)$. Each spectrum represents a specific dose starting from pristine graphene. The inset shows a zoom on the O $1 s$ core level component. Upon irradiation, the $\mathrm{N} 1 s$ line vanishes and the $\mathrm{O} 1 s$ core level line is shifted to lower binding energy, implying a change of the chemical environment of the oxygen atoms.

The flux and flux densities presented in the following are calculated according to the beamline characteristics from ref. [16] The fifth spectrum from the bottom shows the PE survey spectrum after approximately two hours further irradiation at 450 and $800 \mathrm{eV}$ photon energy. All features of the spectrum can be ascribed to either iridium or carbon and are in agreement with the literature $[13,17,18]$. At around 410 and $530 \mathrm{eV}$ binding energy the $\mathrm{N} 1 s$ and $\mathrm{O} 1 s$ core level lines appear upon dosage of $\mathrm{NO}_{2}$. The core level lines reflect the increase of the amount of adsorbed $\mathrm{NO}_{2}$ as expected. The $\mathrm{O} 1 s$ and $\mathrm{N} 1 s$ levels show a ratio of approx. 4:1, which corresponds to a ratio of two oxygen atoms per nitrogen atom, taking into account the photoemission cross sections for the nitrogen and oxygen $1 s$ lines excited by $800 \mathrm{eV}$ photons [19]. After exposure of the sample to light of photon energies between 450 and $800 \mathrm{eV}$ (last spectrum in the row after a total dose of approx. $10^{15}$ photons) the $\mathrm{N} 1 s$ core level component almost completely vanishes, while the $\mathrm{O} 1 s$ core level is shifted to lower binding energies. We assign this fact to a breakup of the adsorbed molecule, with concomitant nitrogen desorption. The shift of the $\mathrm{O} 1 \mathrm{~s}$ core level line between the fourth and fifth spectrum implies a change of the chemical environment of the oxygen atom from adsorbed $\mathrm{NO}_{2}$. We interpret this as a decomposition of $\mathrm{NO}_{2}$, leaving atomic oxygen as a reactant on the surface. This decomposition is apparently induced by the soft x-rays, not unexpectedly since the decomposition of $\mathrm{NO}_{2}$ in the gas phase starts already at $400 \mathrm{~nm}$ (3 eV photon energy) $[10,11]$ and adsorbed on surfaces at $193 \mathrm{~nm}$ or $6.5 \mathrm{eV}$ photon energy [20], but may be also induced by secondary electron-induced decomposition.

Evidence for a chemical reaction of graphene with the oxygen atoms present on the surface can be found in the photoemission data from the graphene/ $\operatorname{Ir}(111)$ substrate. Consider the $\mathrm{C} 1 s$ core level line in Figure 2 and the $\operatorname{Ir} 4 f_{7 / 2}$ core level line in Figure 3. In Figure 2 the formerly sharp C $1 s$ core level line (see the inset) exhibits an additional shoulder approx. $500 \mathrm{meV}$ towards higher binding energy, and a new feature at $286.2 \mathrm{eV}$

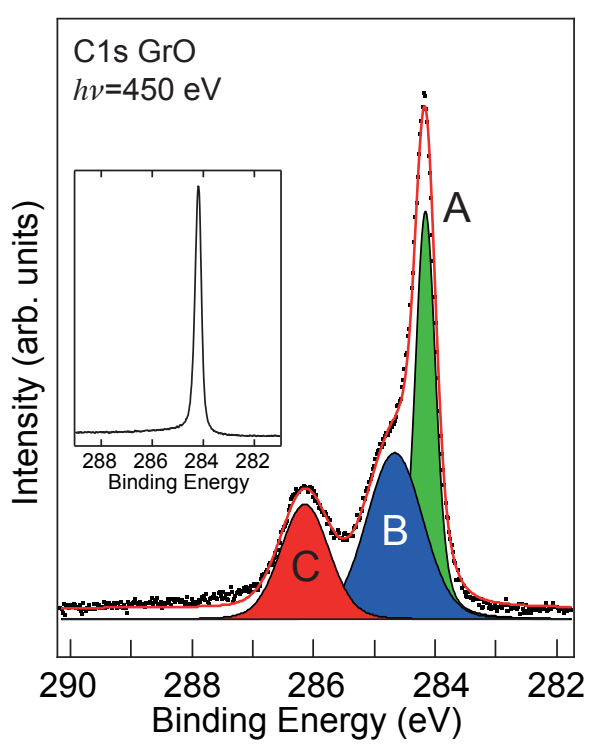

Figure 2: C $1 s$ core level photoemission spectrum for graphene oxide. The components of the fit are (A) $\mathrm{sp}^{2}$-bonded carbon from graphene, (B) $\mathrm{sp}^{3}$ rehybridized carbon neigbouring the epoxidic carbon atoms and $(\mathrm{C})$ epoxidic carbon atoms. The inset shows the pristine graphene/Ir(111) $\mathrm{C} 1 s$ core level line.

binding energy upon $\mathrm{NO}_{2}$ adsorption. This component is usually ascribed to graphene oxide [13, 21-23]. In fact, it is possible to assign specific adsorption sites of the oxygen atoms to the core level line components [13]. In bulk graphite oxide, different oxygen adsorption sites may occur: Hydroxyl groups $(\mathrm{C}-\mathrm{OH})$, ketons $(\mathrm{C}=\mathrm{O})$, esters $(\mathrm{C}-\mathrm{O}-\mathrm{C}$ without a $\mathrm{C}-\mathrm{C}$ bond $)$ and epoxidic oxygen (C-O-C with a $\mathrm{C}-\mathrm{C}$ bond) $[14,24]$. In principle these functional groups also appear in graphene oxide sheets $[22,23]$. In a recent study of oxidized graphene prepared by atomic oxygen exposure, Vinogradov et al. interpreted their findings in terms of mostly epoxidic oxygen formation on graphene/ $\operatorname{Ir}(111)$ [13]. Our observation of two new $\mathrm{C} 1 s$ line components, one at $286.2 \mathrm{eV}$ binding energy and a shoulder around $500 \mathrm{meV}$ higher binding energy apart from the main graphene $\mathrm{C} 1 s$ peak, matches perfectly with their analysis of the formation of an epoxidic oxygen moiety on graphene. The shoulder at $284.6 \mathrm{eV}$ binding energy is assigned to the carbon atoms adjacent to the epoxidic carbon atoms. Due to rehybridization, these neigbouring atoms are now able to bond more strongly to the $\operatorname{Ir}(111)$ substrate, e.g. are pinned (following the nomenclature of Feibelman in ref. [25]) to the surface iridium atoms $[13,25]$. From the best fit of the $C 1 s$ core level lines, using a polynomial background and Voigt profiles with restricted Lorenzian weights, we are able to quantify the amount of oxidized carbon atoms on the surface. The intensity of the pristine $\mathrm{C} 1 \mathrm{~s}$ line is split into roughly $38 \%$ remaining $\mathrm{sp}^{2}$ carbon, $23 \%$ epoxidic carbon and $39 \% \mathrm{sp}^{3}$ hybridized carbon atoms in the oxidized spectrum. Note the FWHM for components B and $\mathrm{C}$ are equal but differ for the $\mathrm{sp}^{2}$ component. This may be caused by structural distortions related to the oxidation and rehybridization. The ratio between the oxidized carbon atoms and the distorted $\mathrm{sp}^{3}$-carbon atoms can be explained by a simple picture: If one $\mathrm{C}=\mathrm{C}$ double bond is oxidized, the two affected 


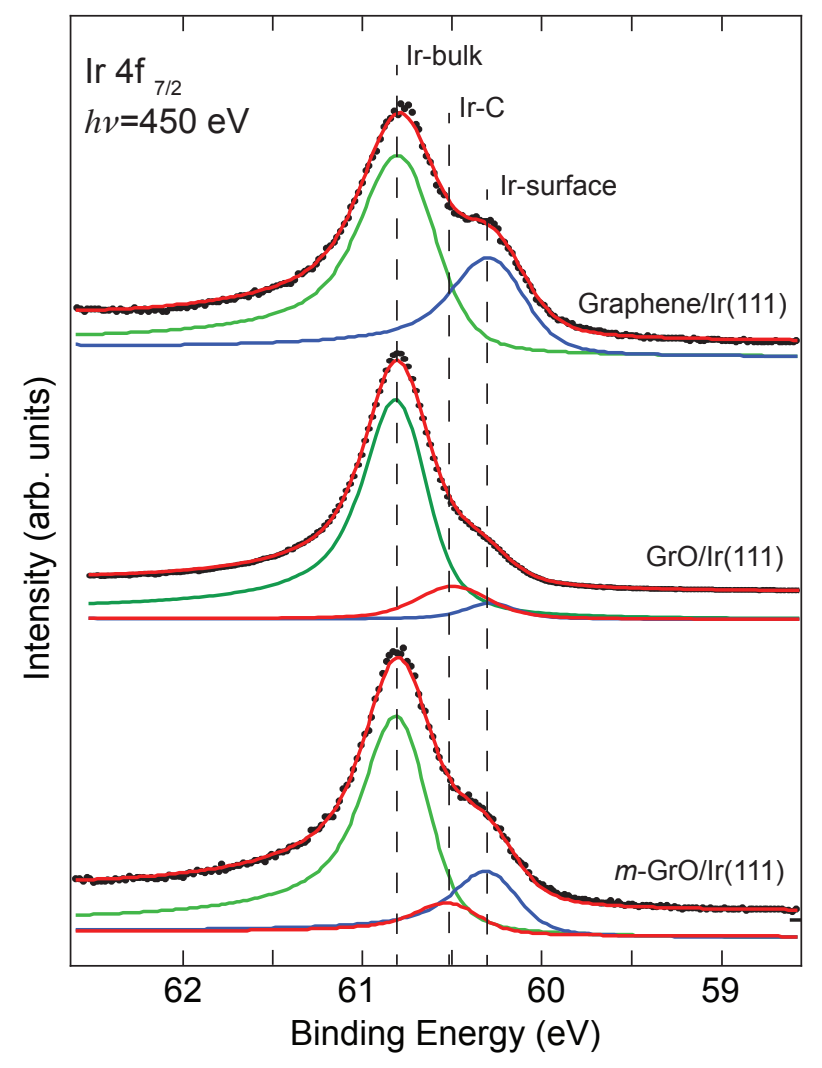

Figure 3: PE spectra from the $\operatorname{Ir} 4 f_{7 / 2}$ core level as observed for pristine graphene/Ir(111), completely oxidized graphene oxide (GrO) and metastable graphene/Ir(111) ( $m$-GrO). The fitted spectral lines are the $\mathrm{Ir} 4 f$ bulk component (green), the surface component (blue) and the Ir-carbon signal (red).

carbon atoms are surrounded by four neigbouring carbon atoms which are distorted. Such a 2:1 ratio (which would be the upper limit for isolated epoxides) is close to the ratio of $1.7: 1 \mathrm{ob}-$ served in our spectra. We thus conclude that $\mathrm{NO}_{2}$ adsorption with subsequent dissociation leads to an oxidized graphene surface in which the oxygen atoms form an epoxidic structure. A small discrepancy of $2 \%$ from the carbon $1 s$ intensity between the fit and the GrO C 1s line is found around $287.5 \mathrm{eV}$ binding energy. This difference may occur from the fitting procedure but may also be related to a missing component. However, an extra component may be resulting from small contaminations at defects, which would in this case not affect the interpretation of the epoxidic component since it is very small.

The bonding of the $\mathrm{sp}^{3}$-distorted carbon atoms also leads to a change in the appearance of the $\operatorname{Ir} 4 f$ core level lines, as shown in Figure 3 for the $\operatorname{Ir} 4 f_{7 / 2}$ core level lines of the pristine graphene/Ir(111) case and the oxidized graphene overlayer. The fit has been performed over the full $\operatorname{Ir} 4 f$ core level line, by using a linear background and spin-orbit split and Doniach-Sunjic corrected Voigt profiles [26]. The $\operatorname{Ir} 4 f$ bulk $\left(60.8 \mathrm{eV} E_{B}\right)$ and surface $\left(60.35 \mathrm{eV} E_{B}\right)$ components are clearly visible. The ratio of these two spectral components results from the probing depth at a photon energy of $450 \mathrm{eV}$ and represents an unperturbed $\operatorname{Ir}(111)$ surface $[13,18]$. Upon oxidation, the Ir $4 f$ surface component is suppressed and partly replaced by an Ir-carbon (Ir-C)

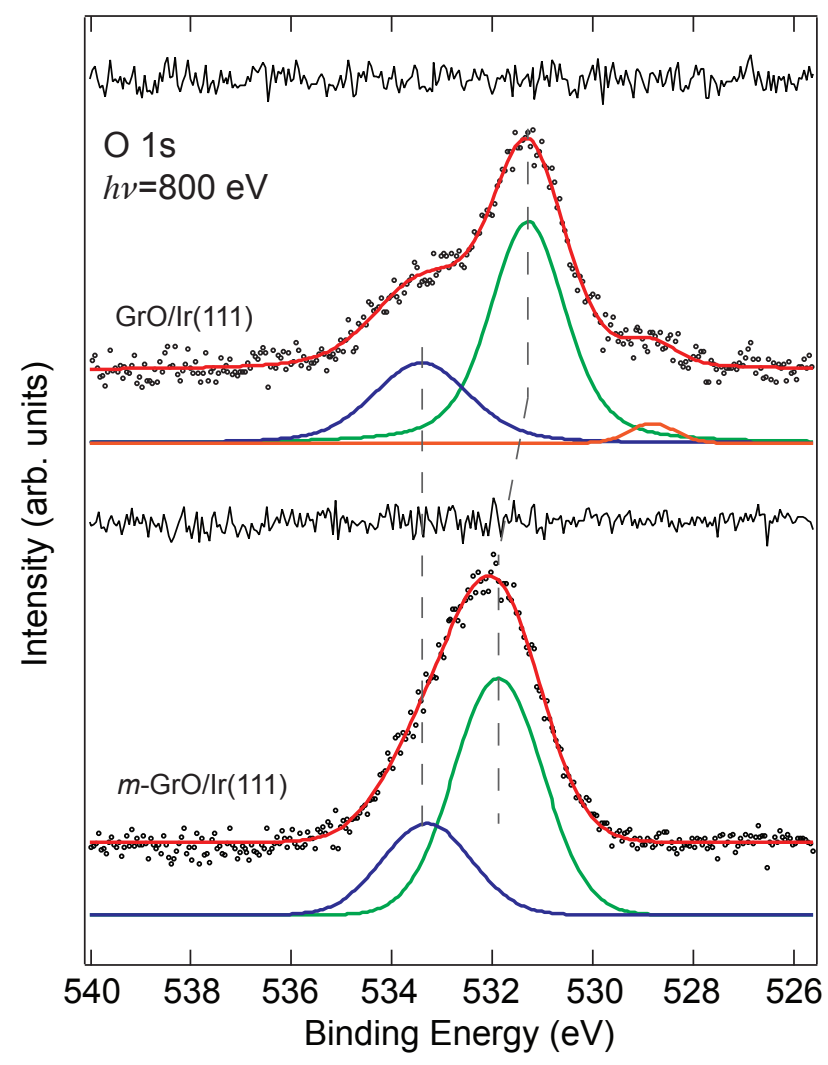

Figure 4: PE spectra from the O1s core for the fully oxidized graphene-oxide $(\mathrm{GrO})$ and metastable graphene-oxide $(\mathrm{m}$-GrO). The fitted spectral lines correspond to epoxidic oxygen (green) intercalated oxygen (orange) and remaining $\mathrm{NO}_{2}$ (blue). The epoxidic line is shifted by approx. $600 \mathrm{meV}$ to higher $\mathrm{BE}$ for $\mathrm{m}-\mathrm{GrO}$.

component. This Ir-carbon line reflects the interaction of the $\mathrm{sp}^{3}$ distorted carbon atoms with the iridium substrate [13].

These observations in general agree with graphene oxide prepared at RT by oxygen atom deposition. However, they let us conclude, especially from the $\mathrm{C} 1 s$ core level line, a high selectivity to epoxidic groups in the prepared $\mathrm{GrO}$ sheet upon irradiation of pre-adsorbed $\mathrm{NO}_{2}$ on graphene/ $\operatorname{Ir}(111)$ and, as we will show later, also fewer defects. The $f c c$ and/or hcp sites are preferred for several adsorbates on graphene moiré structures, e.g. on $\mathrm{Ru}[27], \mathrm{Rh}[28,29]$ and also $\operatorname{Ir}(111)$ $[25,30,31] . \quad \mathrm{NO}_{2}$ probably adsorbs on these $f c c$ or $h c p$ sites. With the loss of nitrogen upon photodissociation of $\mathrm{NO}_{2}$, active oxygen atoms may be formed on the surface only where $\mathrm{NO}_{2}$ is pre-adsorbed, leading to an oxidation specifically located in the hollow sites of graphene/ $\operatorname{Ir}(111)$.

Complementary to the substrate lines, the adsorbate core level spectra give insight into the bonding situation. Figure 4 shows a PE spectrum of the oxygen $1 s$ core level line taken at $800 \mathrm{eV}$ photon energy. The line can be fitted by one main component at $531.3 \mathrm{eV}$ binding energy, i.e. one chemical environment of the oxygen atoms, and two small peaks on either side: one at $528.8 \mathrm{eV}$ binding energy is assigned to intercalated oxygen as found in graphene oxide [13] and oxygen intercalation studies [32, 33]. Oxygen 


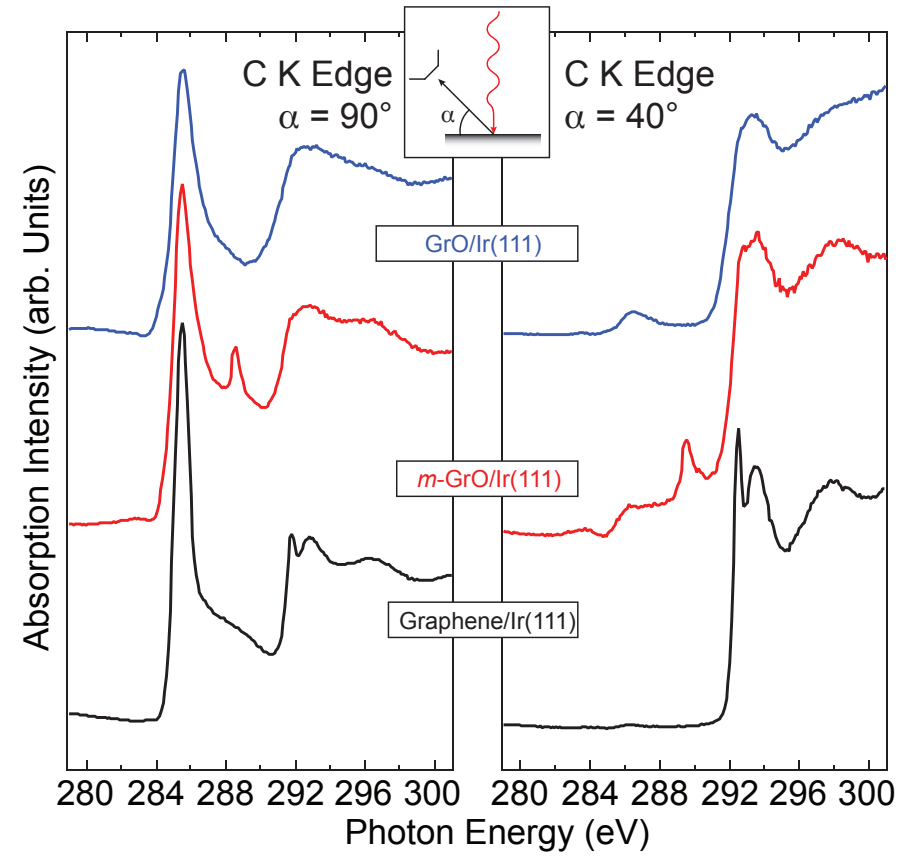

Figure 5: NEXAFS spectra for graphene oxide (blue), metastable graphene oxide (red) and pristine graphene/Ir(111) (black), taken at the carbon $K$ absorption edge. The spectra are collected for normal incident and partially grazing incident light. All spectra are aligned with respect to their $\mathrm{C} 1 s \rightarrow \pi^{*}$ transition.

intercalation is likely to take place at defect sites where the active oxygen species can penetrate the graphene layer, as in other intercalation systems. The second minor component is probably due to remaining $\mathrm{NO}_{2}$, located at $533.4 \mathrm{eV}$ binding energy. At room temperature, this component vanishes. These spectra agree with the observation of Vinogradov et al. [13].

Complementary data for the influence of oxygen on the electronic structure of graphene/ $\operatorname{Ir}(111)$ are derived from the unoccupied valence band states, determined from NEXAFS data on graphene oxidation shown in Figure 5. The spectra for the pristine graphene/ $\operatorname{Ir}(111)$ consist of two main absorption regions, showing the $\mathrm{C} 1 s \rightarrow \pi^{*}$ transitions starting at $285 \mathrm{eV}$ photon energy and the $\mathrm{C} 1 s \rightarrow \sigma^{*}$ transitions, starting from $290 \mathrm{eV}$ photon energy. All spectra are recorded for two different experimental geometries for the incident light beam (light with normal incidence at $\alpha=40^{\circ}$ and for moderate grazing incidence at $\alpha=90^{\circ}$, see inset of Figure 5) in order to assign possible orientations and, to some extent, possible changes in the corrugation of graphene. Due to the nearly perfectly flat graphene layer on $\operatorname{Ir}(111)$ (a height modulation of about $0.3 \AA$ was determined by N'Diyae and coworkers from scanning tunneling microscopy experiments [30]), its $\pi^{*}$ states are almost totally suppressed when the light beam impinges at normal incidence, because the electric field vector of the light in this geometry is perpendicular to the orientation of the $\pi^{*}$ states, while the cross section for the $\sigma^{*}$ states is at its maximum in this configuration [6, 34-36]. Changing the experimental geometry from normal to grazing incidence reverses the effect. The NEXAFS data in Figure 5 show that when
$\mathrm{NO}_{2}$ is adsorbed and irradiated by UV light, the $\mathrm{C} 1 s \rightarrow \pi^{*}$ transition is broadened and the intensity is weaker compared to the pristine case. In addition, the $\mathrm{C} 1 s \rightarrow \sigma^{*}$ regime is also broadened; however, the intensity is almost the same as for the clean surface. This can be explained by rehybridization and the resulting rearrangement of the probed carbon states. When changing the corrugation or the hybridization state of the carbon atoms, the distribution of the $\pi^{*}$-states will differ from the initial upright orientation; by going from $\mathrm{sp}^{2}$ to $\mathrm{sp}^{3}$, the former planar arrangement of the carbon atoms is partially converted into a distorted tetrahedral structure. This leads to a smaller variation of the resonance intensity as a function of angle of incidence [37]. As a consequence, at angles close to normal incidence, $\mathrm{C} 1 s \rightarrow \pi^{*}$ absorption intensity should now also occur and is indeed observed. Furthermore, the intensity for the $\mathrm{C} 1 s \rightarrow \pi^{*}$ transition in grazing incidence should be weaker, as found in our spectra. Although the areas unperturbed by adsorbed oxygen atoms mask these effects to some extent, they are, however, clearly detectable.

Our NEXAFS data show that, upon oxidation of graphene, the electronic structure changes significantly in the range of the low energy unoccupied states. The PE data discussed above imply a change of the graphene surface towards epoxidic structures by chemisorption of oxygen atoms. In addition, NEXAFS shows evidence for a rehybridization of the carbon atoms in the vicinity of the chemisorbed oxygen atoms. This is bound to have an influence on the long range order of graphene. Evidence for this assumption is found in the occupied valence band states of graphene, as shown by valence band photoemission spectra for graphene/ $\operatorname{Ir}(111)$ (black) and graphene-oxide/Ir(111) (red) in Figure 6, taken around the $\Gamma$ point of the hexagonal Brillouin zone. The clean graphene/Ir(111) spectra show the Rashba-split iridium surface state near the Fermi energy [38] as well the intense iridium $4 d$ states around 2-4 eV binding energy and the graphene $\pi$ band around $8 \mathrm{eV}$ binding energy. When graphene is oxidized by irradiation of pre-adsorbed $\mathrm{NO}_{2}$, the intensity of the graphene $\pi$ band is drastically reduced. This can be ascribed to the fact that upon chemisorption and rehybridization towards $\mathrm{sp}^{3}$, the extended electronic structure of graphene is perturbed. In addition, the Ir surface state loses its well-defined shape and the splitting, another indicator for the enhanced bonding of the carbon atoms adjacent to the epoxidic oxygen adsorbates in the graphene layer, as revealed by the changes in the Ir $4 f$ core level states. This observation is consistent with a recent study on band gap formation upon oxidation of graphene/Ir(111) by oxygen atom impact[39]. The authors report a broadening of the graphene $\pi$-band around the $K$ point and an opening of a band-gap at the Dirac point. The broadening is consistent with the depression and a broadening of the $\pi$-band. The Formation of a gap opening is also observed in our experiment, however not presented here.

The formation of graphene oxide from dissociated $\mathrm{NO}_{2}$ is stable at room temperature; the $\mathrm{C} 1 s$ core level, upon warming up to room temperature, shows the same feature at $286.2 \mathrm{eV}$ 


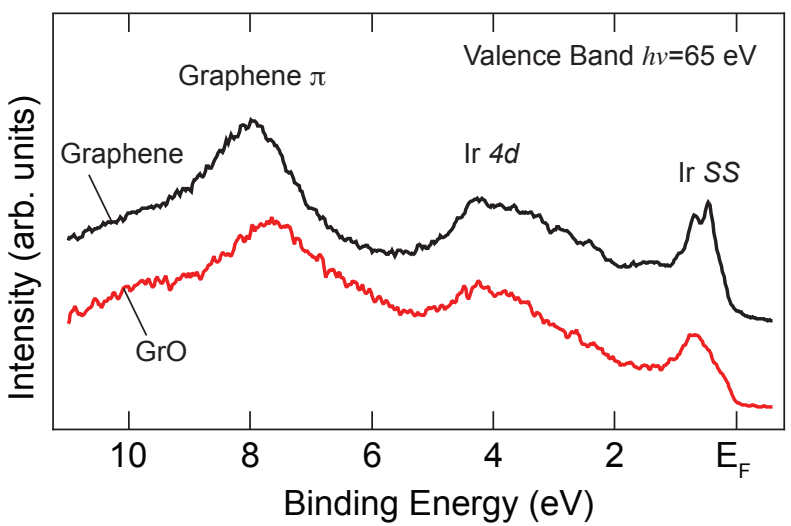

Figure 6: Valence band PES for graphene (black) and graphene oxide (GrO) taken at the $\Gamma$-Point of the hexagonal Brillouin zone. The main features are labeled. Upon oxidation, the graphene $\pi$ band is suppressed and the Ir surface state (Ir $S S$ ) is partly destroyed.

and $284.6 \mathrm{eV}$ binding energy, as for low temperatures, and the Ir $4 f$ core level lines lack their surface components. The $\mathrm{O} 1 \mathrm{~s}$ core level only shows the epoxidic and intercalated species at RT. Only if the sample is annealed to around $500-600^{\circ} \mathrm{C}$, graphene is almost completely restored. A small shoulder remains at the $\mathrm{C} 1 s$ core level at slightly higher binding energies. This was reported to originate from oxygen induced defects, formed during the reduction process [13], as described in the literature [21], supporting our assumption of a reversible photo-oxidation of graphene. However, compared to other methods, the preparation method for graphene oxide presented here shows fewer defects after annealing of graphene, as shown by the $\mathrm{C} 1 s$ core level line and a lower concentration of intercalated oxygen. The method of $\mathrm{NO}_{2}$ adsorption and photon-induced dissociation is likely to be milder than the deposition of oxygen atoms through an atom source which dissociates oxygen molecules by heating up to $1650^{\circ} \mathrm{C}$. This method has been reported to lead to the creation of a high concentration of defects [13]. Our conclusion is also supported by the fact that we observe, in the $\mathrm{O} 1 s$ core level, a small component from intercalated oxygen, which is weaker compared to the study where the atomic oxygen source was used [13].

So far we have investigated the chemical reaction of $\mathrm{NO}_{2}$ with graphene under irradiation of light with a relatively high flux of high energy photons. At lower flux densities another pathway of the adsorption/reaction takes place, which leads to a metastable phase that is hence more difficult to characterize. Flux was reduced in different sets of experiments by changing the so-called $c_{\mathrm{ff}}$ parameter of the x-ray monochromator, which increases the spectral resolution at the expense of flux [40]. In the present case, the $c_{\mathrm{ff}}$ value for the monochromator refraction index (given by the angle between the grating and the mirror) was chosen to change between 2.25 and 8 , resulting in a flux reduction of about one order of magnitude for the latter value.

Figure 7 presents the PE C $1 s$ spectrum recorded from adsorbed $\mathrm{NO}_{2}$ taken at $c_{\mathrm{ff}}=8$. The carbon $1 s$ core level component has now split into multiple components and the fit reveals two

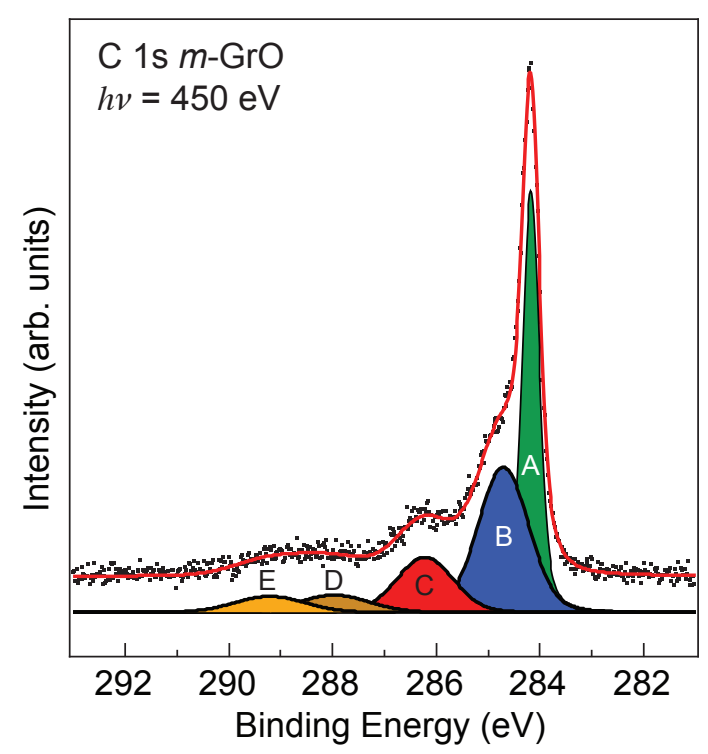

Figure 7: C $1 s$ core level photoemission spectrum for metastable graphene oxide. The components of the fit are (A) $\mathrm{sp}^{2}$-carbon from graphene, (B) $\mathrm{sp}^{3}$ rehybridized carbon neigbouring the epoxidic carbon atoms and (C) epoxidic carbon atoms. The new features (D) and (E) correspond to carbonyl carbon atoms, probably located at the atop and hollow sites of graphene/ $\operatorname{Ir}(111)$

new spectral features (D and E) which appear at binding energies of $288 \mathrm{eV}$ and $289 \mathrm{eV}$ as compared to the spectrum in Figure 2. We believe that these two different features belong to new carbon-oxygen species (probably $\mathrm{C}=\mathrm{O}[22,23]$ ) on both high symmetry areas (the hollow and atop sites) of graphene/Ir(111). This new phase will be denoted as $m$-GrO, where $m$ stands for metastable. From the NEXAFS spectra we assign this phase to a carbonyl (or keton) oxide phase. The NEXAFS spectra in Figure 5 for the metastable phase shows a new, sharp transition feature around $288.5 \mathrm{eV}$ photon energy, found for $\mathrm{C}=\mathrm{O}$ species in graphene-oxide [41] or adsorbed carbon monoxide on metaloxide surfaces [42]. In addition, a strong disturbance in the $\mathrm{sp}^{2}$ character can be observed, especially for normal incidence, visible as a strong background in the energy range of $285-290 \mathrm{eV}$. We assign this effect to the formation of $\mathrm{C}=\mathrm{O}$ double bonds. Upon formation of epoxides, the former $\mathrm{C}=\mathrm{C}$ double bond still exists as a single bond. In order to introduce a $\mathrm{C}=\mathrm{O}$ double bond into the graphene lattice, a $\mathrm{C}=\mathrm{C}$ double bond has to be broken, which leads to a strong disturbance in the lattice. In both cases an $\mathrm{Ir}-\mathrm{C}$ bond may be formed, but the strain within the graphene lattice, consisting of $\mathrm{C}=\mathrm{O}$ double bonds, might be much stronger due to steric considerations, such that the carbon atom environment is heavily distorted. This can explain the strong (and compared to GrO even stronger) rehybridization, observed in $m$-GrO NEXAFS and the larger ratio between peak $\mathrm{B}$ and $\mathrm{C}$ in Figure 7 compared to pure $\mathrm{GrO}$ from Figure 2. The corresponding Ir $4 f$ core level is shown in Figure 3. It shows, apart from the bulk signal, a weakened Ir $4 f$ surface component and a weak Ir-carbon signal. The Ir surface component is not as strongly reduced as for the GrO case above. This means that the Ir-C signature has also not yet developed as strongly as for the fully oxidized case. However, the effect is 
clearly observed and supports our interpretation of the formation of a different oxide formation in $m$-GrO. An analysis of the oxygen $1 s$ core level permits a conclusive picture. The main component is located at $531.9 \mathrm{eV}$ binding energy and is shifted by about $600 \mathrm{meV}$ to higher binding energy compared to GrO. The second component, propably $\mathrm{NO}_{2}$, is located close to the value, observed for GrO. Although this system has to contain carboyl species in principle (see C $1 s$ and NEXAFS data), it is not clearly resolved in the $\mathrm{O} 1 s$. The reason might be its broad lineshape. Although on graphen oxide carbonyl groups are expected at lower BE than the epoxide carbon atoms, [43], $\mathrm{CO}$ adsorbed on $\operatorname{Ir}(111)$ is found to show a core level line at $532.4 \mathrm{eV}$ [44]. The strong rehybridization may lead to a strong shift of the oxygen $1 \mathrm{~s}$ level for the different species. The main component (green) of $m$-GrO has a larger FWHM than the corresponding $\mathrm{GrO}$ line. This may cover different structures of oxidized carbon atoms, inside the oxygen $1 \mathrm{~s}$ line.

There are other possibilities to explain the above observations, such as physisorbed $\mathrm{C}=\mathrm{O}$ on $\mathrm{GrO} / \mathrm{Ir}$ and/or graphene $/ \operatorname{Ir}(111)$ instead of $\mathrm{C}=\mathrm{O}$ groups incorporated into the graphene overlayer. Adsorbed $\mathrm{C}=\mathrm{O}$ would be a simpler explanation and would also lead to the new spectral feature in NEXAFS and might also not be resolved in the $\mathrm{O} 1 s$ beside and epoxide, due to the low coverage. An argument for this interpretation is the fact that the $\mathrm{C}=\mathrm{O}$ signature is lost at room temperature. However, we believe that the $\mathrm{C}=\mathrm{O}$ groups are incorporated in the graphene overlayer for the following reasons: The NEXAFS data show a much stronger disturbance at the $\mathrm{sp}^{2} / \mathrm{sp}^{3}$ state region in the proposed $m$-GrO, which we cannot explain if incorporated $\mathrm{C}=\mathrm{O}$ is absent. The new feature shows no strong angular dependence as expected for an upright oriented $\mathrm{CO}$ molecule. $\mathrm{CO}$ should not be visible in its upright orientation at normal incident light [45]. Also, the ratio between peaks $\mathrm{B}$ and $\mathrm{C}$ in Figure 7 is larger compared to fully oxidized $\mathrm{GrO}$, which let us assume a larger amount of $\mathrm{sp}^{3}$ hybridized carbon atoms compared to the existing epoxidic species. Although $\mathrm{C}=\mathrm{O}$ is still an $\mathrm{sp}^{2}$ carbon species, the lattice strain of $\mathrm{C}=\mathrm{O}$ in the graphene lattice distorts more surrounding carbon atoms as an epoxide. We may speculate that both cases can occur: $\mathrm{C}=\mathrm{O}$ is incorporated into the graphene lattice by the photodissociation of $\mathrm{NO}_{2}$, which leads to a strongly disturbed graphene lattice that is, hence, more active and $\mathrm{CO}$ adsorption could take place as a consequence of the $\mathrm{C}=\mathrm{O}$ incorporation.

The results show that by reducing the photon flux towards $\mathrm{NO}_{2}$ photodissociation, the outcome of the adsorption process can lead to different species being present on the surface. The metastable character of this phase should be emphasized. Apart from the fact that the carbonyl phase is not stable up to room temperature (in contrast to the epoxidic case), it is also possible to drive the reaction towards the stable GrO-phase. Upon irradiation of the $m$-GrO-sample with light of $450 \mathrm{eV}$ photon energy under an equilibrium pressure of $\mathrm{NO}_{2}$ of $5 \times 10^{-8} \mathrm{mbar}$ and an average flux-density of roughly $3 \cdot 10^{18}$ photons $/ \mathrm{s} \cdot \mathrm{cm}^{2}$ (Figure 8), the ratio between the $\mathrm{sp}^{3}$ signal and the epoxidic signal decreases, ending up in the already observed saturated case, which we believe to be the oxidation of the hollow sites with epoxidic graphene-oxide sites as seen in the experiments
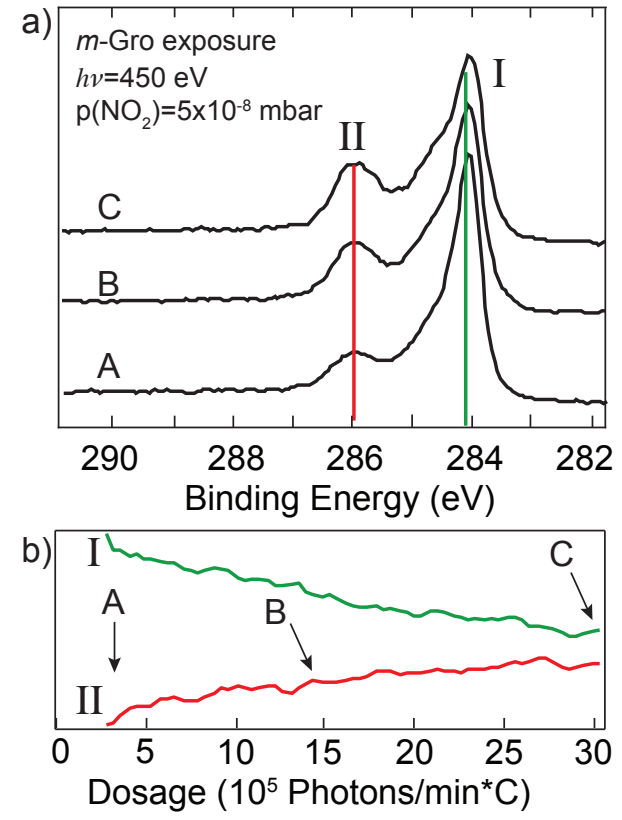

Figure 8: Transitions from the carbon 1s core level line to GrO: Intensity versus exposure of approx. $1 \times 10^{5}$ photons/(min.carbon-atom), with equilibrium pressure of $5 \times 10^{-8} \mathrm{mbar} \mathrm{NO}_{2}$, starting with $m$-GrO. The upper panel a) shows representative extracted spectra at the beginning of the exposure (A at $5 \mathrm{~min}$ ) in the middle ( $\mathrm{B}$ at $15 \mathrm{~min}$ ) and at the end in saturation $(\mathrm{C}$ at $30 \mathrm{~min})$. The lower panel b) shows the evolution of the $\mathrm{sp}^{2}$ carbon component (green line, label I) and the epoxidic carbon component (red line, label II), during the oxidation from $m$-GrO towards GrO.

described at the beginning of this section.

\section{Conclusion}

We report the formation of graphene oxide from adsorbed $\mathrm{NO}_{2}$ on graphene/Ir(111). Dissociation induced by light leads to the formation of active oxygen sites, and under loss of nitrogen, enables the oxygen species to react with the graphene layer. The outcome is an oxidation which we believe to be highly selective at the hollow sites on graphene/ $\operatorname{Ir}(111)$. Furthermore, the oxidation is self-limited to the pre-adsorption sites, which leads to a lower saturation coverage compared to other studies [13]. GrO is stable up to room temperature and can be almost completely converted back into graphene at elevated temperatures. The oxidation reaction is a photon induced process, either direct photodissociation or dissociation from secondary electrons. Beyond the stable $\mathrm{GrO}$ phase, under lower flux densities a metastable graphene-oxide phase appears at low temperature, and a carbonyl species is observed under these conditions. This influence on the rehybridization is even stronger compared to the stable GrO. However, this phase is unstable at room temperature and transforms into stable GrO upon further irradiation/oxidation.

\section{Acknowledgment}

We would like to acknowledge BESSY II and support through the European Science Foundation under the EURO- 
CORES "Euro GRAPHENE" program, project SpinGraph, the Deutsche Forschungsgemeinschaft (DFG) through the Schwerpunktprogramm 1459 "Graphene", and projects HO797/18-1 and DE1679/2-1.

[1] K. Novoselov, A. Geim, S. Morozov, D. Jiang, Y. Zhang, S. Dubonos, I. Grigorieva, A. Firsov, Science 306 (2004) 666.

[2] K. Novoselov, A. Geim, S. Morozov, D. Jiang, Nature 48 (2005) 197.

[3] A. K. Geim, K. S. Novoselov, Nature Mater. 6 (2007) 183.

[4] A. K. Geim, S. V. Morozov, E. W. Hill, M. I. Katsnelson, K. S. Novoselov, Nature Mater. 6 (2007) 652.

[5] K. S. Novoselov, V. I. Fal, L. Colombo, P. R. Gellert, J. Fabian, Nature 490 (2012).

[6] S. Böttcher, M. Weser, Y. S. Dedkov, K. Horn, E. Voloshina, B. Paulus, Nanoscale Res. Lett. 6 (2011) 214.

[7] M. Batzill, Surf. Sci. Rep. 67 (2012) 83.

[8] E. Grånäs, J. Knudsen, U. A. Schröder, T. Gerber, C. Busse, M. A. Arman, K. Schulte, J. N. Andersen, T. Michely, ACS Nano 6 (2012) 9951.

[9] M. Burghard, Surface Science Reports 58 (2005) 1.

[10] I. T. N. Jones, J. Chem. Phys. 59 (1973) 4836.

[11] E. P. Gardner, P. D. Sperry, J. G. Calvert, J. of Geophys. Res.: Atmospheres 92 (1987) 6642.

[12] J. N. Crowley, S. A. Carl, J. Phys. Chem. A 101 (1997) 4178

[13] N. A. Vinogradov, K. Schulte, M. L. Ng, A. Mikkelsen, E. Lundgren, N. Mårtensson, A. B. Preobrajenski, J. Phys. Chem. C 115 (2011) 9568.

[14] Y. Zhu, S. Murali, W. Cai, X. Li, J. W. Suk, J. R. Potts, R. S. Ruoff, Adv. Mater. 22 (2010) 3906.

[15] R. Larciprete, S. Fabris, T. Sun, P. Lacovig, A. Baraldi, S. Lizzit, J. Am Chem. Soc. 133 (2011) 17315.

[16] W. Mahler, unpublished, 2012.

[17] C. Busse, P. Lazić, R. Djemour, J. Coraux, T. Gerber, N. Atodiresei, V. Caciuc, R. Brako, A. T. N'Diaye, S. Blügel, J. Zegenhagen, T. Michely, Phys. Rev. Lett. 107 (2011) 036101

[18] J. F. Van der Veen, F. J. Himpsel, D. E. Eastman, Phys. Rev. Lett. 44 (1980) 189

[19] J. J. Yeh, I. Lindau, Atomic data and nuclear data tables 32 (1985) 1.

[20] K. Sawabe, Y. Matsumoto, Chem. Phys. Lett. 194 (1992) 45.

[21] M. Z. Hossain, J. E. Johns, K. H. Bevan, H. J. Karmel, Y. T. Liang, S. Yoshimoto, K. Mukai, T. Koitaya, J. Yoshinobu, M. Kawai, A. M. Lear, L. L. Kesmodel, S. L. Tait, M. C. Hersam, Nature Chem. 4 (2012) 305.

[22] J. Liu, S. Fu, B. Yuan, Y. Li, Z. Deng, J. Am. Chem. Soc. 132 (2010) 7279

[23] L. Q. Xu, W. J. Yang, K.-G. Neoh, E.-T. Kang, G. D. Fu, Macromol 43 (2010) 8336.

[24] K. A. Mkhoyan, A. W. Contryman, J. Silcox, D. A. Stewart, G. Eda, C. Mattevi, S. Miller, M. Chhowalla, Nano Lett. 9 (2009) 1058.

[25] P. Feibelman, Physical Review B 77 (2008) 165419.

[26] S. Doniach, M. Sunjic, Journal of Physics C: Solid State Physics 3 (1970) 285.

[27] Z. Zhou, F. Gao, D. W. Goodman, Surf. Sci. 604 (2010) L31.

[28] E. Voloshina, Y. S. Dedkov, S. Torbrügge, A. Thissen, M. Fonin, Appl. Phys. Lett. 100 (2012) 241606.

[29] M. Sicot, S. Bouvron, O. Zander, U. Rüdiger, Y. S. Dedkov, M. Fonin, Appl. Phys. Lett. 96 (2010) 093115.

[30] A. N'diaye, S. Bleikamp, P. Feibelman, T. Michely, Phys. Rev. Lett. 97 (2006) 215501.

[31] E. Voloshina, E. Fertitta, A. Garhofer, F. Mittendorfer, M. Fonin, A. Thissen, Y. S. Dedkov, Sci. Rep. 3 (2013) 1

[32] H. Zhang, Q. Fu, Y. Cui, D. Tan, X. Bao, J. Phys. Chem. C 113 (2009) 8296.

[33] M. Ostler, R. J. Koch, F. Speck, F. Fromm, H. Vita, M. Hundhausen, K. Horn, T. Seyller, Mater. Sci. Forum 717-720 (2012) 649.

[34] Y. S. Dedkov, M. Fonin, New J. Phys. 12 (2010) 125004.

[35] Y. S. Dedkov, M. Sicot, M. Fonin, Journal Of Applied Physics 107 (2010) 09E121.

[36] M. Weser, E. Voloshina, K. Horn, Y. S. Dedkov, Phys. Chem. Chem. Phys. 13 (2011) 7534.

[37] J. Stöhr, D. A. Outka, Phys. Rev. B 36 (1987) 7891.

[38] A. Varykhalov, D. Marchenko, M. Scholz, Phys. Rev. Lett. 108 (2012) 066804.

[39] K. Schulte, N. A. Vinogradov, M. L. Ng, N. Mårtensson, A. B. Preobra- jenski, App. Surf. Sci. 267 (2013) 74.

[40] H. Petersen, C. Jung, C. Hellwig, W. B. Peatman, W. Gudat, Rev. Sci. Instrum. 66 (1995) 1.

[41] S. Dubin, S. Gilje, K. Wang, V. C. Tung, K. Cha, A. S. Hall, J. Farrar, R. Varshneya, Y. Yang, R. B. Kaner, ACS Nano 4 (2010) 3845.

[42] R. Lindsay, A. Gutierrez-Sosa, G. Thornton, A. Ludviksson, S. Parker, C. T. Campbell, Surf. Sci. 439 (1999) 131.

[43] A. Barinov, O. B. Malcioğlu, S. Fabris, T. Sun, L. Gregoratti, M. Dalmiglio, M. Kiskinova, J. Phys. Chem. C 113 (2009) 9009.

[44] T. Fujitani, I. Nakamura, Y. Kobayashi, A. Takahashi, M. Haneda, H. Hamada, Journal Of Physical Chemistry B 109 (2005) 17603.

[45] J. Stohr, K. Baberschke, R. Jaeger, R. Treichler, S. Brennan, Phys. Rev. Lett. 47 (1981) 381 